\title{
Hyperbaric oxygen effects on the alveoli-capillary unit in a murine model of pulmonary arterial hypertension
}

Pedro J. Curi-Curi ${ }^{1}$, Maria C. Castillo-Hernandez ${ }^{1}$, Miguel Chavez-Martinez ${ }^{2}$, Lilia Loredo-Mendoza ${ }^{3}$, Eduardo Rios-Garcia ${ }^{1}$, Alexandre Kormanovski-Kovzova ${ }^{1}$, Gustavo Guevara-Balcazar ${ }^{1}$

${ }^{1}$ Escuela Superior de Medicina, Instituto Politecnico Nacional, Mexico ${ }^{2}$ Department of Pathological Anatomy, Hospital Regional de Alta Especialidad de Ixtapaluca, Mexico

${ }^{3}$ Histology Department, Universidad Panamericana, Mexico

Submitted: 6 April 2020

Accepted: 16 August 2020

Arch Med Sci

DOI: https://doi.org/10.5114/aoms.2020.98985

Copyright $\odot 2020$ Termedia \& Banach

\section{Abstract}

Introduction: Pulmonary hypertension $(\mathrm{PH})$ is an increase in the normal value of systolic pressure of the pulmonary artery, frequently due to congenital heart diseases being its treatment the correction of this defects; alternatively, hyperbaric oxygen (HBO) has shown beneficial effects facilitating neovascularisation and decreased vascular resistance. An experimental study was designed to determine the effect of HBO on the alveoli-capillary unit in a murine model of irreversible pulmonary hypertension (IPAH) induced with monocrotaline.

Material and methods: Four groups of 10 Wistar rats each were considered: A (control), B (HBO), C (IPAH), and D (IPAH+HBO). HBO was administered at $2 \mathrm{~atm}, 1 \mathrm{~h}$ daily, for 15 days. At the end of this time, systolic pulmonary artery pressure (PAP) was measured, followed by histologically quantification of the number of cells, vascular buttons, and neoformation vessels employing the immunochemistry detection of hypoxia-induced factor-1 (HIF-1), vascular endothelial growth factor (VEGF), and CD34, respectively.

Results: Histologically, this matches with an increase of the average number of positive HIF-1 cells, and an increase in VEGF-positive vascular buttons in group $D$ rats compared with those of group $C$. The number of CD34-positive neovessels was similar in groups $C$ and $D$, but in the latter group the number of capillary vessels was more significant.

Conclusions: This suggests that angiogenesis and vasculogenesis were induced by HIF-1, and it has been achieved due to an early effect of HBO therapy. Presumably, neovessels were formed between days 5 and 10 following HBO therapy, but more experimental studies are required to test this hypothesis.

Key words: hyperbaric therapy, pulmonary arterial hypertension, monocrotaline.

\section{Introduction}

Pulmonary hypertension $(\mathrm{PH})$ is defined as a mean pulmonary artery pressure $(P A P)>25 \mathrm{~mm} \mathrm{Hg}$ at rest beyond the first few months of life or $>30 \mathrm{~mm} \mathrm{Hg}$ with exercise in an adult person [1]. In the paediatric population, $\mathrm{PH}$ is usually considered as a systolic pulmonary artery pressure (PAP) similar to or higher than the systolic systemic arterial one. This haemodynamic condition results from pulmonary vascular disease (PVD)

\author{
Corresponding author: \\ Gustavo Guevara-Balcazar \\ $\mathrm{MD}, \mathrm{PhD}$ \\ Escuela Superior \\ de Medicina \\ Instituto Politecnico Nacional \\ Mexico \\ E-mail: \\ castillohernandezmc@gmail. \\ com
}


and includes $\mathrm{PH}$ related to cardiac, lung, and systemic diseases, as well as idiopathic pulmonary artery hypertension [2].

The current clinical classification of $\mathrm{PH}$ [3] shows five large groups according to its cause. The first of these groups include the most frequent aetiologies at paediatric stage, which are congenital heart diseases with left-to-right shunt and increased pulmonary overload, which leads to pre-capillary antegrade $\mathrm{PH}$ [4]. Heath and Edwards developed a histopathological classification of $\mathrm{PH}$ [5] in six grades, of which the last three usually show irreversible tissue damage and clinically present as Eisenmenger syndrome [6]. This syndrome is characterised by the installation of cardiopulmonary failure and right-to-left (inverted) shunting, with subsequent clinical onset of cyanosis. This clinical scenario translates an obstructive pulmonary vascular disease that appears in the terminal stages of congenital heart disease with left-to-right shunting, left to its natural history in the absence of treatment, and is considered as a prelude to multiorgan failure and death. When a congenital heart disease progresses to this stage, it resembles that of a terminal metastatic cancer for which current conventional and advanced therapeutic measures are useless, and the prognosis is poor [7].

On the other hand, hyperbaric oxygen therapy (HBO), consists of exposure to a partial pressure of $\mathrm{O}_{2}$ higher than $1 \mathrm{~atm}$ [8]. HBO stimulates angiogenesis, increases cardiac output, increases blood flow in ischaemic tissues, diminishes reperfusion damage [9], and inhibits the reproduction of anaerobic and micro-aerophilic microorganisms [10, 11]. Although HBO's benefits in wound healing have been documented, the mechanism of action by which it does this continues to be investigated and appears to be a combination of systemic events as well as local alterations within the margin of the wound [12]. It can be highlighted that the final effects related to the improvement of tissue survival in ischaemia are mainly due to neovascularisation. This phenomenon occurs through two processes. The first, angiogenesis [13], is the regional stimulus that increases the outbreak of blood vessels from the growth of local endothelial cells (vascular buttons), where vascular endothelial growth factor (VEGF) plays an important role. The second is vasculogenesis [14], which is the stimulation of recruitment and the differentiation of circulating stem or progenitor cells to form new vessels (neovessels). Despite the HBO effects on the mobilisation of stem cells mediated by oxidative stress [15], the production of the VEGF in the sites of neovascularisation is stimulated. This is due, at least in part, to the synthesis and stabilisation of hypoxia-inducible elements (HIF) [16]. These tran- scription factors are heterodimers of HIF- $\alpha$, and a constitutively expressed HIF- $\beta$. The expression and activation of HIF- $\alpha$ subunits are importantly regulated as well as their degradation via the ubiquitin-proteasome, which usually occurs when the cells are full of $\mathrm{O}_{2}$ [17]. However, whether there are hypoxic or normoxic prevalent conditions, free radicals are required for HIF expression [18]. HBO raises levels of HIF-1 and HIF-2 in CD34-positive vasculogenic progenitor or stem cells due to an increase in reactive oxygen species (ROS).

The goal of the present study was to evaluate HBO morpho-functional effects on the alveoli-capillary unit in an experimental model of Wistar rats with irreversible pulmonary hypertension (IPAH). Endpoints were to demonstrate $\mathrm{HBO}$ capacity to modify PAP levels as well as to influence the histological count of HIF-1 cells, VEGF vascular buttons, and the CD34 positive neovessels in the alveolicapillary units of the rats with IPAH. We hypothesised that systolic pulmonary artery pressure normalises due to an increased number of vessels in the alveoli-capillary unit of the Wistar rats with IPAH in response to $\mathrm{HBO}$ therapy.

\section{Material and methods}

\section{Animals}

Male Wistar rats, weighing 300-400 g, 12 weeks old, were randomly allocated to four groups (10 animals per group): A - without IPAH or HBO therapy (control); $\mathrm{B}$ - without IPAH but with $\mathrm{HBO}$ therapy (sham); $\mathrm{C}-$ with IPAH but without $\mathrm{HBO}$ therapy (non-treated); and D - with IPAH and $\mathrm{HBO}$ therapy (treated). A monocrotaline pharmacological model was used to induce IPAH. The Institutional Animal Care and Use Committee also approved the procedures.

\section{IPAH model}

The IPAH model was based on a standardised pharmacological method [19]. A single $60 \mathrm{mg} / \mathrm{kg}$ dose of Monocrotaline was administered through an abdominal rat subcutaneous injection, waiting up to 14 days for IPAH development.

\section{HBO therapy}

Rats with HBO therapy breathed $100 \%$ oxygen at $2 \mathrm{~atm}$ absolute (ATA) for $1 \mathrm{~h}$ daily for 15 days.

\section{Dissection, PAP measures, and collection of samples}

All rats received PAP measures regardless of their group. After an intraperitoneal anaesthesia with Xylazine $^{\circledast} 0.5 \mathrm{mg} / \mathrm{kg}$ and Ketamine ${ }^{\circledast} 8 \mathrm{mg} / \mathrm{kg}$, orotracheal intubation, and support with mechanical ventilator (Harvard Rodent Ventilator, Model 683, 
$21 \% \mathrm{FiO}_{2}, 56$ to 58 per minute ventilatory rate, and $1.5 \mathrm{ml} / \mathrm{kg}$ current volume), a sub-xiphoid surgical approach was made. The right ventricle was punctured with an insulin needle connected to a catheter and later transduced with a computerised software (BIOPAC MP100). PAP records were digitally stored for a 1-minute lapse of time to calculate an average value for the rats of each group. After PAP measurement, all rats were sacrificed with an overdose of sodium pentobarbital (70 mg/kg), subsequently performing heart and lung block evisceration.

\section{Lung histological and immunochemical analysis}

Samples of all lung tissues (upper, middle, lower and posterior right lung lobes, and single left lung lobe) were stored in $10 \%$ formaldehyde for light microscopy and immunochemistry assessments. Lung tissue for light microscopy analysis was paraffin-embedded, sectioned at the middle part of each lobe, and stained with haematoxylin and eosin. After histological examination to identify the type and predominance of Heath and Edwards grades of $\mathrm{PH}$ in each group, capillary count at a 40x light microscopy was performed in 10 fields, to obtain the mean number of capillaries per area using the stereology method.

After completion of the histological study, three more additional transversal sections in the middle part of the single left lung lobe of each rat were made for immunohistochemical analysis. Images were acquired with a light microscope, and the positive count was made evaluating ten fields at 40x magnification with the stereology technique. A mean positive cell count for 10 microscopic areas was obtained for every rat in the four studied groups. Staining for HIF-1 was used to detect triggering of vasculogenesis and angiogenesis. Staining for VEGF was used to show vascular growth from pre-existing vessels. Staining for CD34 was used to identify newly formed blood vessels.

\section{Statistical analysis}

GraphPad Prism ${ }^{\circledast}$ version 5.01 software was used for statistical analysis. Quantitative data were expressed as a percentage and measurement data as average \pm SD (minimum-maximum range). Comparisons between groups were performed by using one-way analysis of variance (ANOVA), with $p<0.05$ for the difference being considered statistically significant.

\section{Results}

\section{Functional PAP measurement}

Figure 1 shows the registered patterns of transduced PAP and a comparison of mean PAP functional measurement values. Note that the standard high-altitude PAP value was $12.7 \pm 1.2$ $\mathrm{mm} \mathrm{Hg}$ for rats in the control group (A). The only group that reached $\mathrm{PH}$ criteria (mean PAP: 27.8 $\pm 11.2 \mathrm{~mm} \mathrm{Hg}$ ) was the non-treated group (C), which showed 2.2 times higher than normal PAP with statistical significance. When comparing PAP between the treated group (C) with that of the non-treated group (D), there was a statistically significant difference $(p<0.05)$. Additionally, PAP values between the treated group (D) and the control group (A) remained similar $(p>0.05)$.

\section{Lung microscopy}

Histological patterns found in the normal (A) and sham (B) groups were those of reversible $\mathrm{PH}$, with no significant differences in the lobes and lungs distribution. The normal group A showed the same distribution of grade 1 (53\%) and grade $2(47 \%)$ patterns, while group B showed grade 2 (45\%) quite similarly to grade 3 (55\%) pattern findings. On the other hand, the non-treated group (C) and the treated group (D) showed IPAH patterns, without significant differences in the lobe and lung distribution, confirming the systemic monocrotaline damage. Grade 5 (55\%) was predominantly found in the non-treated group (C), followed by grade 4 (25\%) and grade 6 (20\%) histological patterns. The treated group (D) also showed grade 5 (50\%), predominantly histological patterns, followed by grade $6(30 \%)$ and grade 4 (20\%).

Figure 2 shows a representative view that resumes all findings in the four studied groups, regarding the mean number of capillaries counted in 10 microscopic fields of observation at 40x. Comparatively, there are statistically significantly more capillaries in the treated group (D) than in the other three groups (A, B, and C).

\section{Lung immunochemistry}

Immunochemistry count of positive HIF-1 cells, VEGF vascular buttons, and CD34 neoformation vessels were made by determining the mean number obtained at 10 microscopic views with $40 \times$ per rat for all groups.

A microscopic representative pattern for HIF-1 immunochemical staining as well as positive cell count comparison in all groups is represented in Figure 3. Note that the treated group showed a statistically significantly higher cell count than the non-treated group $(p=0.004)$. There are similar numbers of positive HIF-1 cell count in the treated group (D) when compared with the normal one (A).

Figure 4 shows the immunochemical VEGF staining pattern view, as well as the mean pos- 
Pedro J. Curi-Curi, M.C. Castillo-Hernandez, M. Chavez-Martinez, L. Loredo-Mendoza, E. Rios-Garcia, A. Kormanovski-Kovzova, G. Guevara-Balcazar
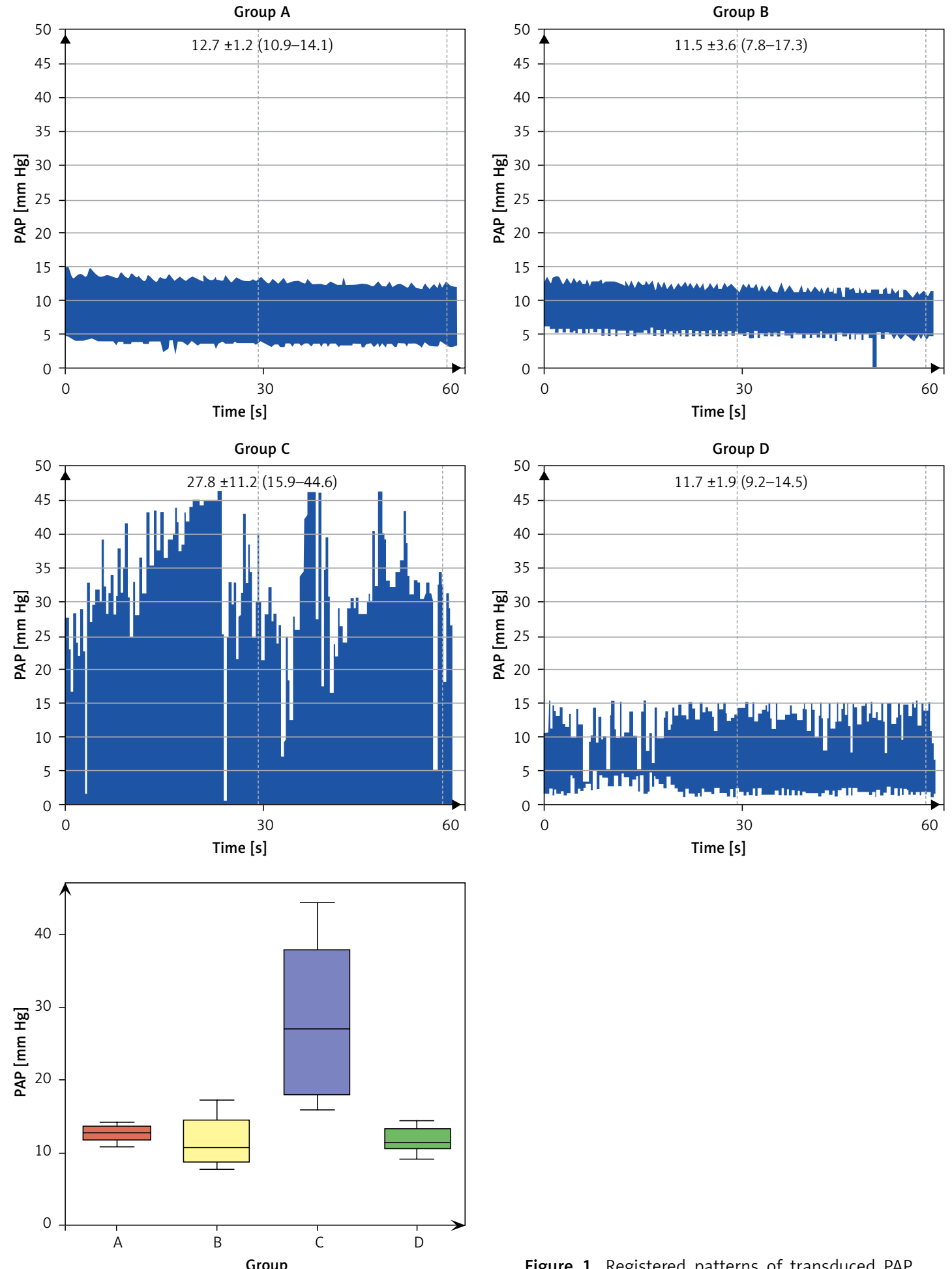

A vs. B $(p=\mathrm{NS}) \quad$ A vs. $\mathrm{C}(p<0.05) \quad$ A vs. D $(p=\mathrm{NS})$ B vs. C $(p<0.05) \quad$ B vs. D $(p=N S) \quad$ C vs. D $(p<0.05)$

Figure 1. Registered patterns of transduced PAP and comparison of mean PAP $(\mathrm{mm} \mathrm{Hg})$ values at the time of rat sacrifice in all groups 
A

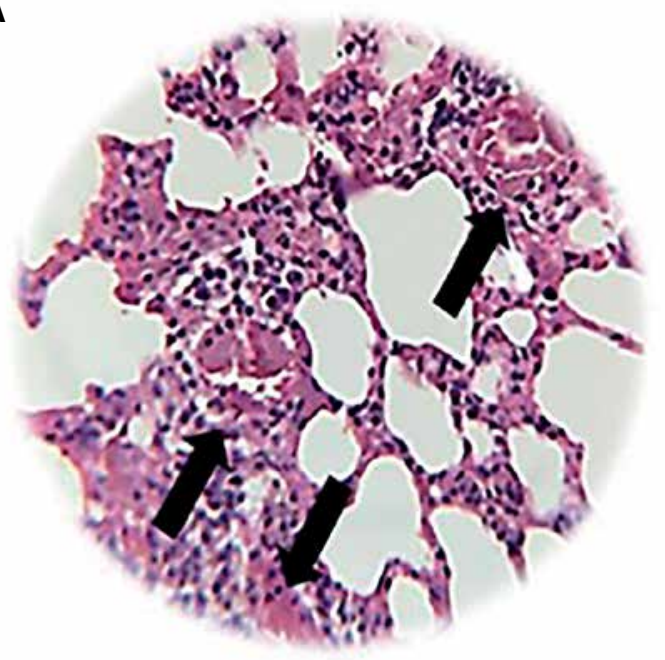

$2.6 \pm 0.8$ capillaries per field (1-4)

c

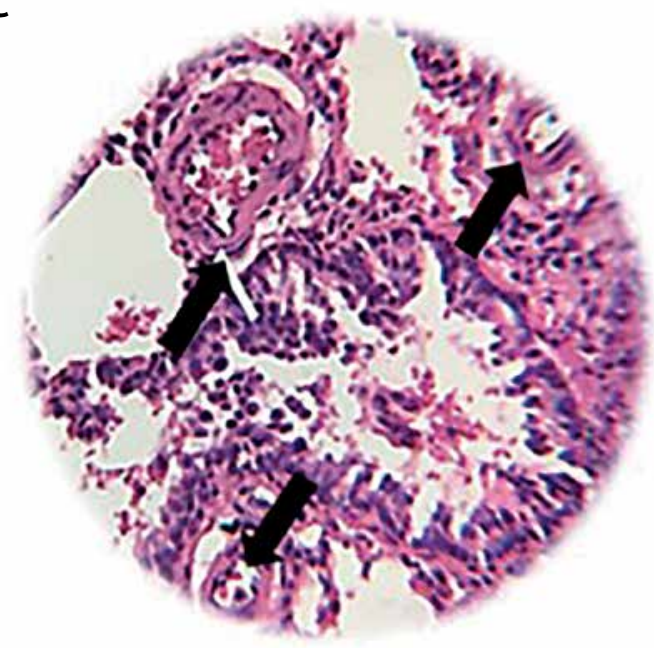

$2.6 \pm 0.5$ capillaries per field $(2-3)$

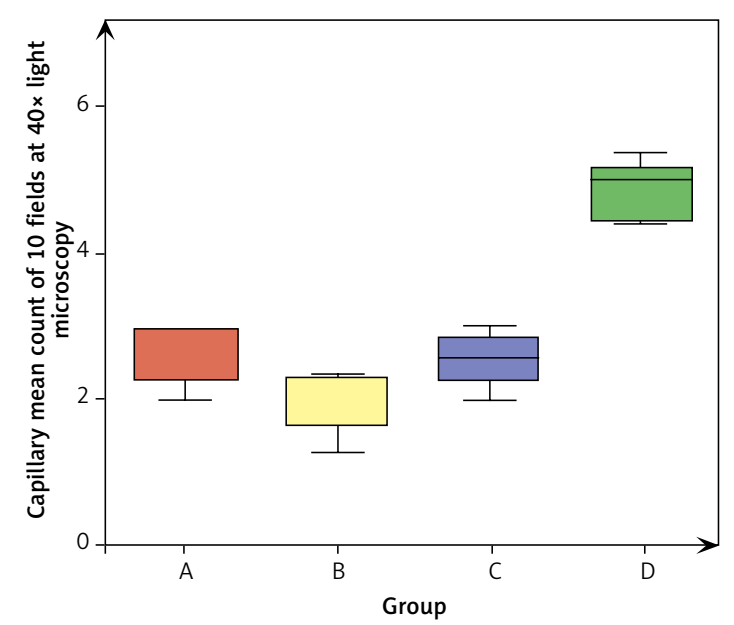

A vs. B $(p=N S) \quad$ A vs. C $(p=N S) \quad$ A vs. $D(p<0.05)$ B vs. C $(p=N S) \quad$ B vs. D $(p<0.05) \quad$ C vs. $\mathrm{D}(p<0.05)$
B

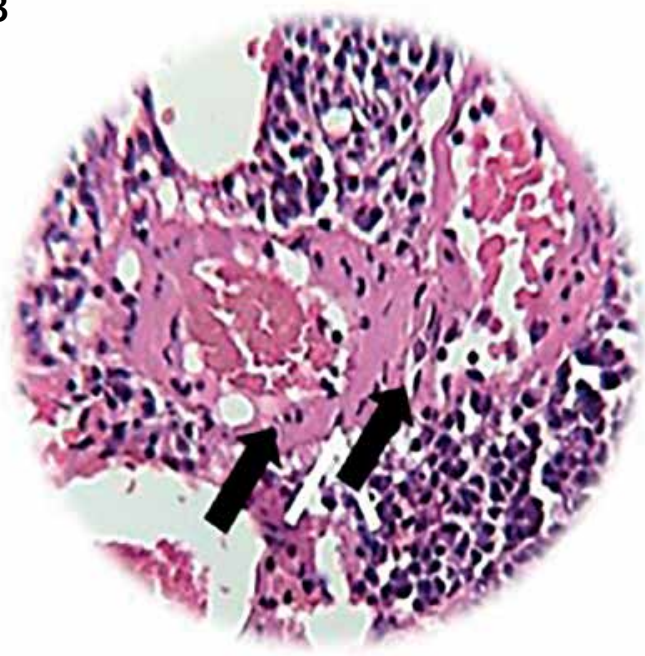

$2 \pm 0.6$ capillaries per field $(1-3)$

D

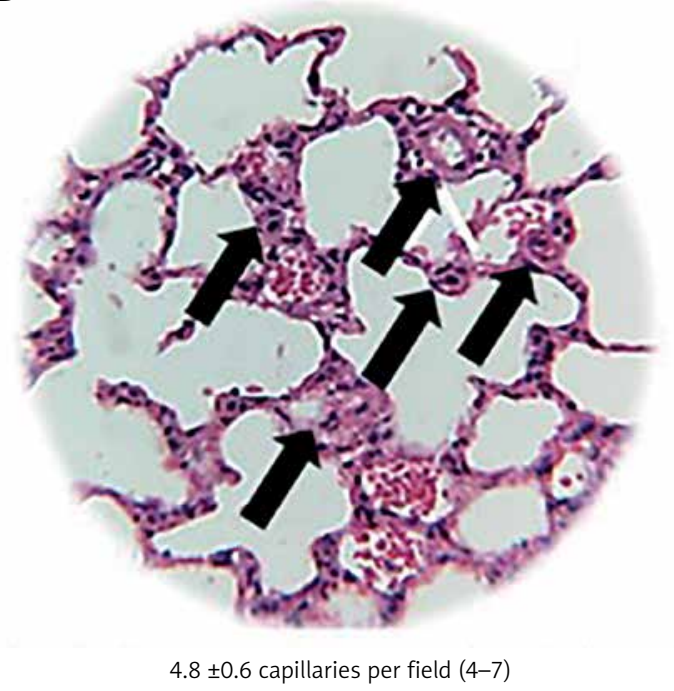

Figure 2. Microscopic view of the mean pulmonary capillary count at 10 observational fields (black arrows show the number of capillaries counted per file) and a comparison between the mean capillary count in all groups 
Pedro J. Curi-Curi, M.C. Castillo-Hernandez, M. Chavez-Martinez, L. Loredo-Mendoza, E. Rios-Garcia, A. Kormanovski-Kovzova, G. Guevara-Balcazar
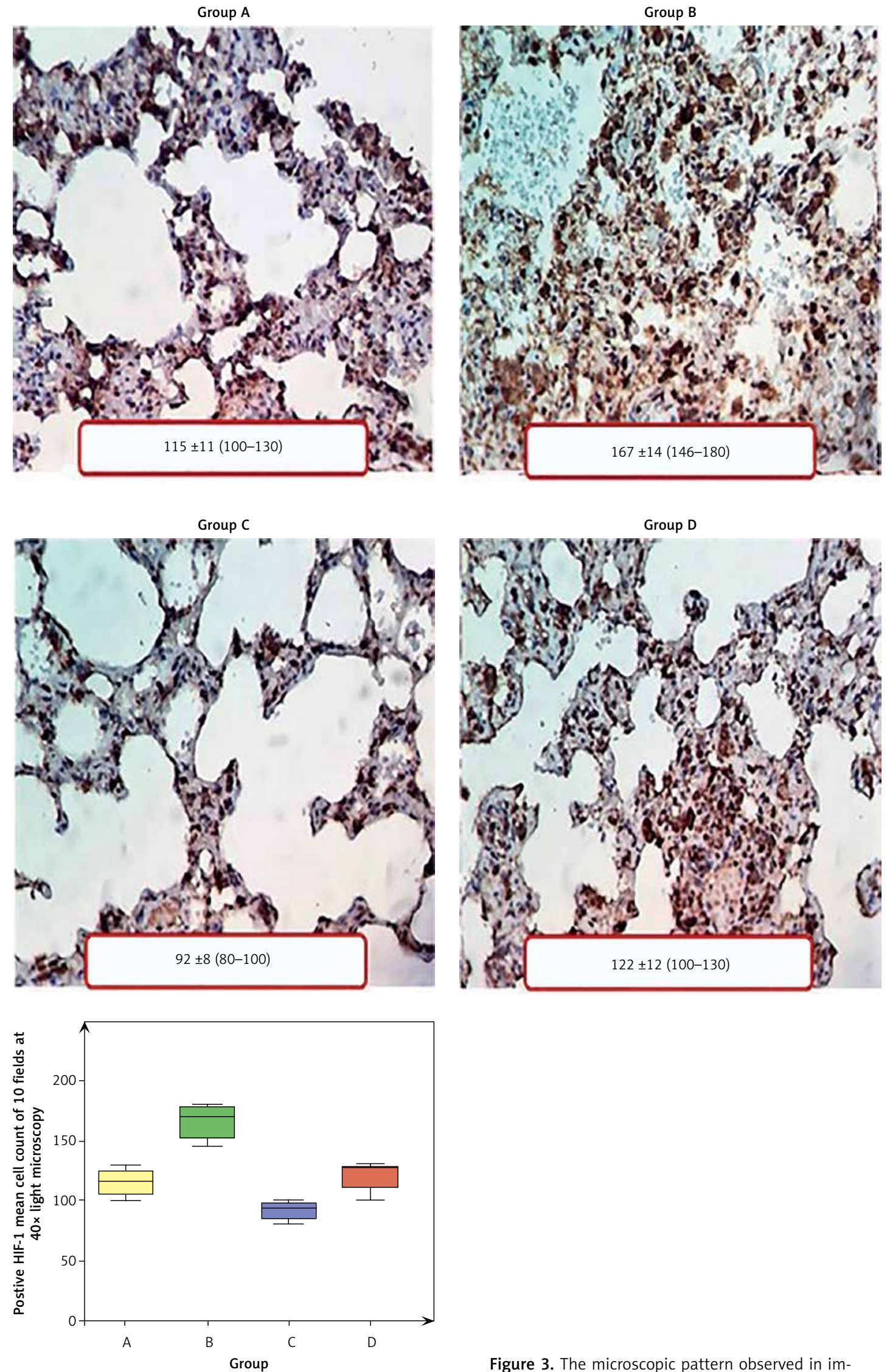

A vs. $\mathrm{B}(p<0.05) \quad$ A vs. C $(p<0.05) \quad$ A vs. D $(p=\mathrm{NS})$

B vs. C $(p<0.05) \quad$ B vs. D $(p<0.05) \quad$ C vs. $\mathrm{D}(p<0.05)$

Figure 3. The microscopic pattern observed in immunochemical HIF-1 staining and mean favourable cell count comparison between all groups 

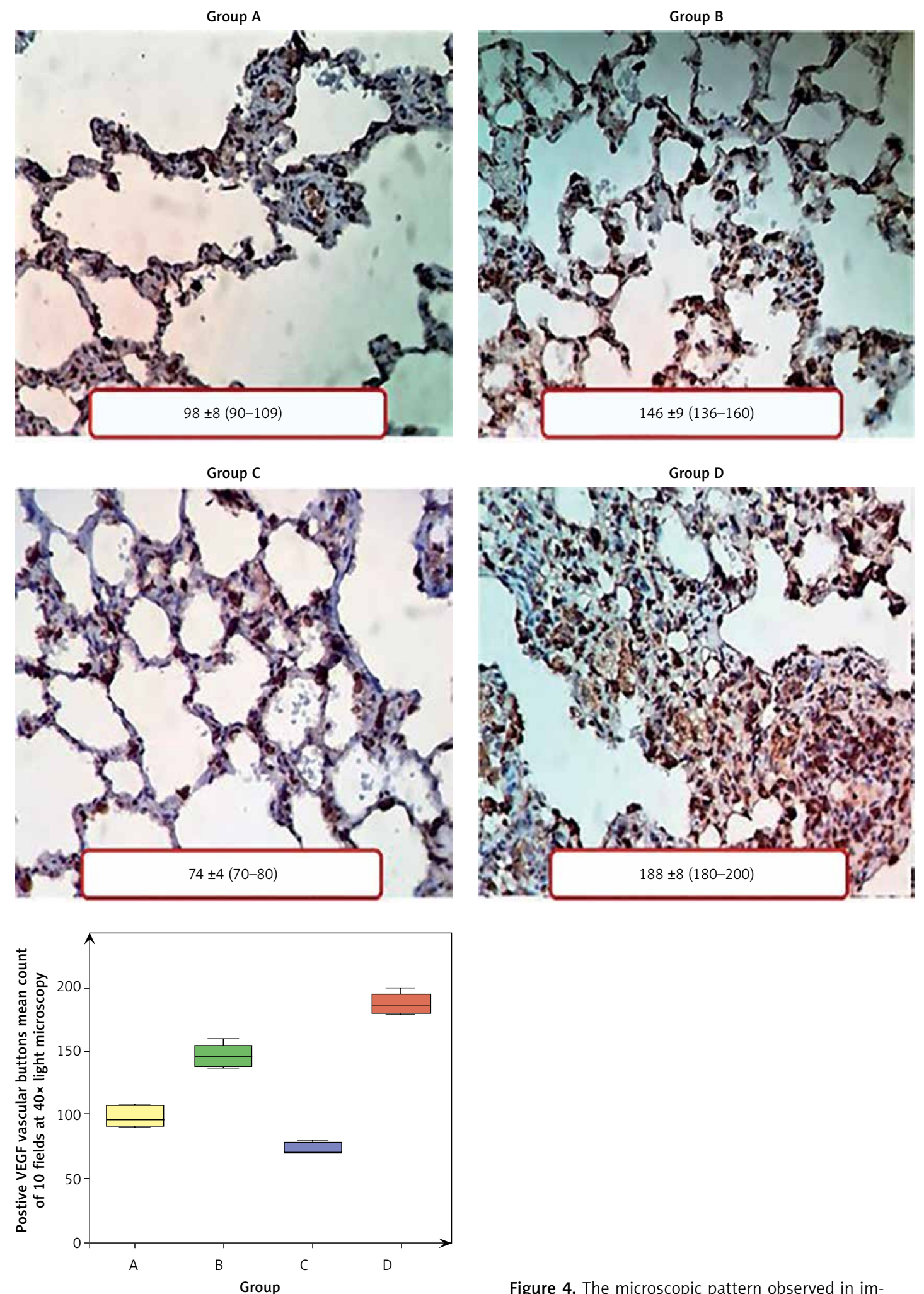

A vs. $\mathrm{B}(p<0.05) \quad$ A vs. $\mathrm{C}(p<0.05) \quad$ A vs. $\mathrm{D}(p<0.05)$ B vs. C $(p<0.05) \quad$ B vs. $D(p<0.05) \quad$ C vs. $D(p<0.05)$

Figure 4. The microscopic pattern observed in immunochemical VEGF staining and mean positive cell count comparison between all groups 
itive vascular button count compared between all groups. There are significantly fewer positive vascular buttons in group C (non-treated) than in group $D$ (treated), which shows a similar count when compared with the normal group (A).

An immunochemical pattern for CD34 neoformation vessel staining and microscopic mean count for positive CD34 neovessels comparison between all groups was also performed (Figure 5). There were a similar number of mean neovessels between the normal group (A), the treated group (D), and the non-treated group (C).

\section{Discussion}

One of the findings to highlight in our research is that, although we expected to have a regular lung histologic pattern in the control group (A), we observed in a considerable percentage of cases, grade $1 \mathrm{PH}$ lesions (55\% of muscular hypertrophy in the right lung and $40 \%$ in the left lung), as well as $\mathrm{PH}$ grade 2 patterns (45\% of intimal proliferation in the right lung and $60 \%$ in the left lung). A probable explanation for this finding is the fact that all the Wistar rats used in this study and included in the control group were born in Mexico City, which has an average altitude of $2240 \mathrm{~m}$ [20]. The effects of living at this altitude are due to the low barometric pressure, which leads to a reduction in the partial oxygen pressure of the inspired air. This condition of hypobaric hypoxia is the cause of alveolar hypoxia and chronic hypoxaemia in humans that live at high altitudes (more than $1500 \mathrm{~m}$ above sea level), or to acute hypoxaemia in those who ascend to these altitudes. In a historical review, Reeves and Grover [21] showed that human newborns at high altitudes, as well as newborns at sea level, have $\mathrm{PH}$ and a thick middle layer of smooth muscle cells in the small pulmonary vessels and arterioles [22], findings similar to those described in the rats in group A of this study. In contrast, in newborns at high altitudes, vascular remodelling occurs slowly during life, and this is the reason for the persistence of primary $\mathrm{PH}$ and right ventricular hypertrophy into adulthood [23].

One of the most critical microscopic findings of this work is the significantly higher increase in the number of lung capillaries in group D (IPAH with HBO) compared to group C (IPAH without HBO). This is related to the decrease in PAP in the treated group (D) compared to the non-treated group (C), reaching values similar to those recorded in the rats of the control group $(A)[24,25]$.

Therefore, because there is a more significant number of vessels, the value of the radius increases considerably, producing a substantial decrease in the pulmonary vascular resistance, and consequently, also in the systolic pressure of the pulmonary artery. This mechanism contrasts with the current therapeutic trend to increase the radius of the pulmonary capillaries employing vasodilator agents to achieve a decrease in vascular resistance and pulmonary arterial hypertension. Additionally, the reduction of $\mathrm{PAH}$ values through increasing the number of pulmonary capillary vessels could be synergised, eventually with the use of vasodilators to achieve an optimal effect in the control of PAP through both mechanisms. In clinical practice, vasodilators such as prostanoids or nitric oxide therapy are already used to decrease $\mathrm{PH}$ through the production of ROS, but their effect can definitely be potentiated with HBO supply in order to improve outcomes in this varied group of patients [26].

Once we clarified how HBO therapy normalises PAP in IPAH, we attempted to elucidate the molecular mechanisms underlying the pathogenesis of $\mathrm{PH}$. Therefore, an immunohistochemical analysis was made, including the essential markers for lung neovascularisation (VEGF and CD34) and the factor that presumably triggers this pathway (HIF-1) using HBO. It has been established that HBO therapy increases the production of ROS, which are a trophic stimulus for bone marrow stem cells [27]. It is also well known that ROS are necessary for the expression of the hypoxia-1 inducible factor (HIF-1) [28]. HIF-1 is a membrane protein that can be found in several organs (heart and lung, among others) and is composed of two subunits: HIF- $1 \alpha$ and HIF- $1 \beta$. In a situation of normoxia, HIF$1 \alpha$ is degraded by hydroxylation and is destroyed by the ubiquitin-proteasome pathway. However, when there is a lack of oxygen, as is the case of hypoxia, HIF- $1 \alpha$ does not degrade but joins HIF- $1 \beta$, leading to HIF-1 formation, which is the main stimulator for the expression of currently more than a dozen different genes regulated by oxygen [29]. One of them is VEGF, which is the most specific factor directly involved in neovascularisation. Neovascularisation occurs by two processes. First, there is the stimulus for the recruitment and differentiation of pluripotent stem cells in the bone marrow, and when released into the circulation they form de novo vessels through a process called vasculogenesis. The second process is the regional stimuli that influence the efficiency of the growth of new blood vessels by local endothelial cells, which is called angiogenesis. HBO therapy has effects on both processes.

Although it is well documented that VEGF plays a crucial role in vasculogenesis [30, 31], there is a scientific lack of information regarding VEGF pathways that induce differentiation of mesodermal progenitor cells to vascular progenitor cells, and then into CD34+ vascular pluripotential cells and CD34- cells. The CD34+ vascular pluripotent cells synthesise several vascular cell markers, as well as proteins related to vasculogenesis and 

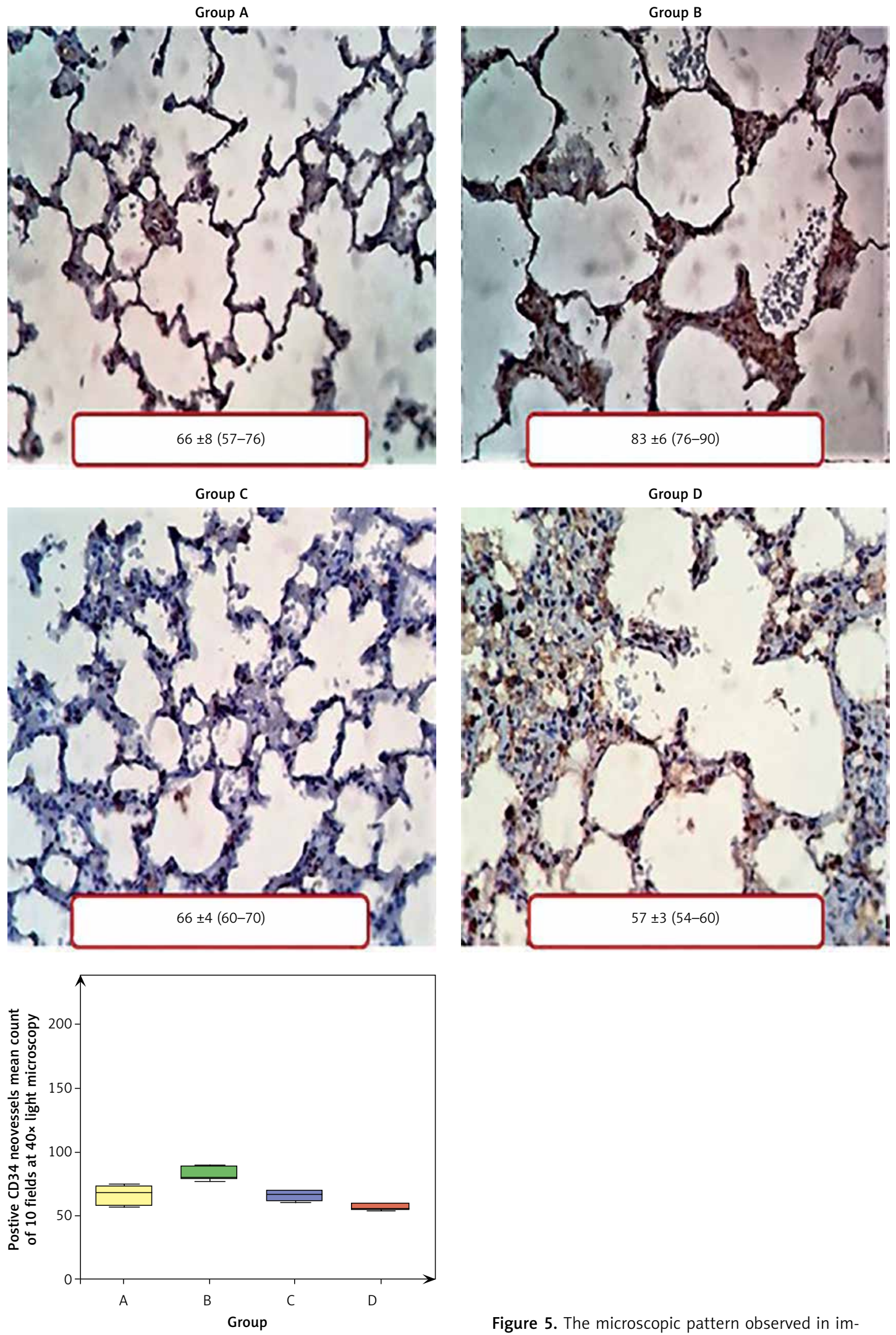

$\begin{array}{lll}\text { A vs. B }(p<0.05) & \text { A vs. C }(p=N S) & \text { A vs. D }(p<0.05) \\ \text { B vs. C }(p<0.05) & \text { B vs. D }(p<0.05) & \text { C vs. D }(p=N S)\end{array}$

Figure 5. The microscopic pattern observed in immunochemical CD34 staining and involve positive cell count comparison between all groups 
smooth muscle differentiation. CD34- cells consist of a mixed population that over-synthesises factors that can promote angiogenesis [32-34].

Immunochemistry of the present study for HIF-1, VEGF, and CD34 at the end of the 15 days of HBO therapy in the treated group (D) suggests the following chronology of events: 1) Release of HIF-1 by $\mathrm{HBO}, 2)$ Expression of VEGF genes induced by HIF-1, and 3) Activation of vasculogenesis and angiogenesis at the tissue level with new formation of capillary vessels. We believe that the release of HIF-1, VEGF, and CD34 have, like most enzymes and organic hormones, a rising curve, a peak, and a decent curve that, presumably can be cyclical as the HBO doses, although the effects that they trigger (like the newly formed vessels) are persistent. By day 15 , which is the moment in which the cross-section was made, a relative decrease in the HIF-1 count, a corresponding increase in the VEGF neovascularisation buttons, and a reduction in CD34 are observed because there is already a more significant number of vessels must have been formed previously. This suggests that angiogenesis and the induced HIF-1 vasculogenesis is achieved due to an early effect of HBO therapy. Presumably, neovessels were formed between days 5 and 10 following HBO therapy, but more experimental studies are required to test this hypothesis.

Some limitations of this study, which become future challenges to be clarified, are the determination of the exact day on which lung vasculogenesis occurs due to HBO, and the elucidation of the effect of this therapy is lasting or is lost when it is discontinued. It must be, as well, crucial to know if we can shorten $\mathrm{HBO}$ therapeutic sessions by increasing $\mathrm{HBO}$ doses or by increasing the atmospheric pressure used.

In conclusion, HBO morpho-functional effects in a murine model of IPAH can be summarised as follows: It diminishes PAP values up to normalisation, increases the number of lung capillaries, increases VEGF vascular buttons as well as HIF-1 positive cell count, and keeps the number of CD34 neovessels without significant changes after 15 days of HBO therapy. Therefore, HBO therapy shows a beneficial effect on the alveolicapillary unit, because it is capable of increasing the number of pulmonary capillaries after 15 days, promoting neovascularisation of the respiratory unit in an experimental model of Wistar rats with IPAH, leading to a decrease and normalisation of PAP levels.

Understanding the favourable functional effects of HBO therapy in terms of PAP normalisation even in the most severe cases of IPAH is also beneficial in the less critical cases where pulmonary vascular resistances are still reversible. HBO can be used as well as an adjuvant therapy to reduce morbidity and mortality, and improve the prognosis of adult $\mathrm{PAH}$ groups such as those with left severe heart disease [35], lung disease, and different $\mathrm{PH}$ scenarios, regardless of its aetiology.

\section{Conflict of interest}

The authors declare no conflict of interest.

\section{References}

1. Galiè N, Humbert M, Vachiery JL, et al. 2015 ESC/ERS Guidelines for the diagnosis and treatment of pulmonary hypertension: The Joint Task Force for the Diagnosis and Treatment of Pulmonary Hypertension of the European Society of Cardiology (ESC) and the European Respiratory Society (ERS): Endorsed by: Association for European Paediatric and Congenital Cardiology (AEPC), International Society for Heart and Lung Transplantation (ISHLT). Eur Heart J 2016; 37: 67-119.

2. Abman SH, Hansmann G, Archer SL, et al. Pediatric Pulmonary Hypertension: Guidelines From the American Heart Association and American Thoracic Society. Circulation 2015; 132: 2037-99.

3. Simonneau G, Gatzoulis MA, Adatia I, et al. Updated clinical classification of pulmonary hypertension. Turk Kardiyol Dern Ars 2014; 42 Suppl 1: 45-54.

4. Ezekian JE, Hill KD. Management of pulmonary arterial hypertension in the pediatric patient. Curr Cardiol Rep 2019; 21: 162.

5. Heath D, Edwards JE. The pathology of hypertensive pulmonary vascular disease: a description of six grades of structural changes in the pulmonary arteries with special reference to congenital cardiac septal defects. Circulation 1958; 18: 533-47.

6. Chaix MA, Gatzoulis MA, Diller GP, Khairy P, Oechslin EN. Eisenmenger syndrome: a multisystem disorder-do not destabilize the balanced but fragile physiology. Can J Cardiol 2019; 35: 1664-74.

7. Gatzoulis MA, Landzberg M, Beghetti M, et al.; MAESTRO Study Investigators. Evaluation of macitentan in patients with Eisenmenger syndrome. Circulation 2019; 139: 51-63.

8. Chu DK, Kim LH, Young PJ, et al. Mortality and morbidity in acutely ill adults treated with liberal versus conservative oxygen therapy (IOTA): a systematic review and meta-analysis. Lancet 2018; 91: 1693-705.

9. Li WF, Huang YQ, Feng YQ. Oxygen therapy for patients with acute myocardial infarction: a meta-analysis of randomized controlled clinical trials. Coronary Artery Dis 2018; 29: 652-6.

10. Gill AL, Bell CN. Hyperbaric oxygen: its uses, mechanisms of action and outcomes. QJM 2004; 97: 385-95.

11. Andrade SM, Santos IC. Hyperbaric oxygen therapy for wound care. Rev Gaucha Enferm 2016; 37: e59257.

12. Thom SR. Hyperbaric oxygen: its mechanisms and efficacy. Plast Reconstr Surg 2011; 127 Suppl 1 (Suppl 1): 131S-41S

13. Huang YJ, Nan GX. Oxidative stress-induced angiogenesis. J Clin Neurosci 2019; 63: 13-6.

14. Ratajska A, Jankowska-Steifer E, Czarnowska E, et al. Vasculogenesis and its cellular therapeutic applications. Cells Tissues Organs 2017; 203: 141-52.

15. Thom SR. Oxidative stress is fundamental to hyperbaric oxygen therapy. J Appl Physiol 2009; 106: 988-95. 
16. Chen L, Endler A, Shibasaki F. Hypoxia and angiogenesis: regulation of hypoxia-inducible factors via novel binding factors. Exp Mol Med 2009; 41: 849-57.

17. Befani C, Liakos P. The role of hypoxia-inducible factor-2 alpha in angiogenesis. J Cell Physiol 2018; 233: 9087 98.

18. Serocki M, Bartoszewska S, Janaszak-Jasiecka A, Ochocka RJ, Collawn JF, Bartoszewski R. miRNAs regulate the HIF switch during hypoxia: a novel therapeutic target. Angiogenesis 2018; 21: 183-202.

19. Gomez-Arroyo JG, Farkas L, Alhussaini AA, et al. The monocrotaline model of pulmonary hypertension in perspective. Am J Physiol. Lung Cell Mol Physiol 2012; 302: L363-9.

20. Menezes AM, Perez-Padilla R, Jardim JR, et al; PLATINO Team. Chronic obstructive pulmonary disease in five Latin American cities (the PLATINO study): a prevalence study. Lancet 2005; 366: 1875-81.

21. Reeves JT, Grover RF. Insights by Peruvian scientists into the pathogenesis of human chronic hypoxic pulmonary hypertension. J Appl Physiol 2005; 98: 384-9.

22. Penaloza D. Efectos de la exposición a grandes alturas en la circulación pulmonar. Rev Española Cardiol 2012; 65: 1075-8.

23. Shosholcheva M, Aankulovski N, Kartalov A, Kuzmanovska B, Miladinova D. Synergistic effect of hyperoxia and biotrauma on ventilator-induced lung injury. Prilozi (Makedonska akademija na naukite i umetnostite. Oddelenie za medicinski nauki) 2017; 38: 91-6.

24. Synolakis CE, Badeer HS. On combining the Bernoulli and Poiseuille equation - a plea to authors of college physics texts. Am J Phys 1989; 57: 1013-9.

25. Gallagher KA, Goldstein LJ, Thom SR, Velazquez OC. Hyperbaric oxygen and bone marrow-derived endothelial progenitor cells in diabetic wound healing. Vascular 2006; 4: 328-37.

26. Jasińska-Stroschein M, Stawarczyk K, Stępień A, Orszulak-Michalak D. Comparative tolerability of targeted therapies in pulmonary hypertension. Arch Med Sci 2020. DOI: https://doi.org/10.5114/aoms.2020.96143.

27. Schroedl C, McClintock DS, Budinger GR, Chandel NS Hypoxic but not anoxic stabilization of HIF-1alpha requires mitochondrial reactive oxygen species. Am J Physiol 2002; 283: L922-31.

28. Welsh SJ, Bellamy WT, Briehl MM, Powis G. The redox protein thioredoxin-1 (Trx-1) increases hypoxia-inducible factor 1alpha protein expression: Trx-1 overexpression results in increased vascular endothelial growth factor production and enhanced tumor angiogenesis. Cancer Res 2002; 62: 5089-95.

29. Chang YN, Zhang K, Hu ZM, et al. Hypoxia-regulated IncRNAs in cancer. Gene 2016; 575: 1-8.

30. Ribatti D. The discovery of the fundamental role of VEGF in the development of the vascular system. Mechanisms Development 2019; 160: 103579.

31. Baghdady Y, Hussein Y, Shehata M. Vascular endothelial growth factor in children with cyanotic and acyanotic and congenital heart disease. Arch Med Sci 2010; 6: 221-5.

32. Marvasti TB, Alibhai FJ, Weisel RD, Li RK. CD34+ stem cells: promising roles in cardiac repair and regeneration. Canad J Cardiol 2019; 35: 1311-21.

33. Chao J, Guo Y, Li P, Chao L. Opposing Effects of oxygen regulation on Kallistatin expression: Kallistatin as a novel mediator of oxygen-induced HIF-1-eNOS-NO pathway. Oxid Med Cell Longev 2017; 2017: 5262958.

34. Abo-Grisha N, Essawy S, Abo-ElMatty D, Abdel-Hady Z. Effects of intravenous human umbilical cord blood
CD34+ stem cell therapy versus levodopa. Arch Med Sci 2013; 9: 1138-51.

35. Mehra P, Mehta V, Sukhija R, et al. Pulmonary hypertension in left heart disease. Arch Med Sci 2019; 15 : 262-73. 